\title{
THE APPLICATION OF DIRECTIONAL UNIVARIATE STRUCTURE FUNCTIONS ANALYSIS FOR STUDYING THE SPATIAL ANISOTROPY OF ENVIRONMENTAL VARIABLES
}

\author{
DARIA SVIDZINSKA
}

Department of Physical Geography and Geoecology, Faculty of Geography, Taras Shevchenko National University of Kyiv, 2A Akademika Glushkova ave., MSP-680, Kyiv, Ukraine; e-mail: d.svidzinska@gmail.com

\begin{abstract}
Svidzinska D.: The application of directional univariate structure functions analysis for studying the spatial anisotropy of environmental variables. Ekológia (Bratislava), Vol. 38, No. 2, p. 140-153, 2019.

As anisotropy is a fundamental property of the real-world environmental spatial variables, the conventional omnidirectional variograms and correlograms do not provide means enough to characterise spatial dependence between observations. The purpose of this article is to introduce directional univariate structure functions analysis to explore and quantify the spatial anisotropy of environmental variables. Analysis of six environmental variables within three physical-geographical regions proved the leading role of relief for landscape differentiation; it also defined the size and extension of major landforms responsible for the organisation of spatial pattern. The arrangement of the vegetation patches demonstrated linkage with the major landforms. The other relief derivatives, being prone to noise and artefacts in the original data, showed a random-variable type of behaviour. In the lack of any particular spatially anisotropic structure, the results of the analysis can provide a clue about meaningful distances of interest at finer scales. The approach can also be an exploratory tool for discrete measurements to recognise the features of spatial continuity.

Key words: autocorrelation, environmental variable, Kanivs'kiy Nature Reserve, semivariance, spatial analysis, spatial anisotropy.
\end{abstract}

\section{Introduction}

Most of the real-world environmental variables are inherently anisotropic, which means that spatial dependence between a variable's values is not the same for all geographic directions considered (Cressie, 1993; Legendre, Legendre, 2012; Rossi et al., 1992). It is important to account for the presence and features of this dependence to understand spatial patterns in the data (Legendre, Fortin, 1989; Rossi et al., 1992; Turner, Gardner, 2015), to catch the scale of data variability (Legendre, Legendre, 2012), to reveal links between variables (Rossi et al., 1992; Turner, Gardner, 2015) and to describe the main features of spatial anisotropy (Rossi et al., 1992). This information is routinely used to hypothesise on the nature and scale of a pattern-generating process (Legendre, Fortin, 1989; Turner, Gardner, 2015) and to develop sampling schemes (Cressie, 1993). 
The tools to characterise spatial dependence between observations are the univariate structure functions of semivariance and autocorrelation (Cressie, 1993; Legendre, Legendre, 2012; Rossi et al., 1992). Both are calculated across a distance, whilst the semivariance is a measure of the variance and the autocorrelation is a measure of the correlation of a regionalised variable (Cressie, 1993; Legendre, Legendre, 2012). When used simultaneously, these functions provide insights on the existence of a spatial pattern in the data, degree of its spatial variability and critical distances at which significant similarities or dissimilarities are observed (Legendre, Fortin, 1989; Rossi et al., 1992; Turner, Gardner, 2015).

Traditionally, a single semivariance or autocorrelation value is calculated for each distance class, producing the so-called omnidirectional variograms and correlograms. But Legendre and Legendre (2012) argued that for spatial environmental variables, when anisotropy is the case, it is necessary to account for a directional change in the values of the univariate structure functions. Whilst the directional univariate structure functions analysis is recognised to be more appropriate for the spatially anisotropic realworld data, its practical implementation may be limited by a small number of irregularly spread observations (Legendre, Legendre, 2012) as well as the lack of ready-to-apply analytical and visualisation techniques (Legendre, Fortin, 1989; Rosenberg, 2000; Rossi et al., 1992).

Here, I describe how to apply the directional univariate structure functions analysis to the real-world environmental data. I examined the set of six raster environmental variables for the three sub-areas of different physical-geographical regions. Using the data, I illustrate how the results can identify and quantify complex spatial patterns and their scales. This, in turn, helped to recognise factors and/or processes responsible for landscape heterogeneity at different scales.

\section{Material and methods}

\section{Study area}

The study area of $1,528 \mathrm{~km}^{2}$ covers the overall extent of the Kanivs'kyi Nature Reserve, Ukraine, and its surroundings, defined by a 2-km buffer zone (Copernicus Programme, 2015; Hansen, DeFries, 2007) (Fig. 1). As a part of the Dnieper River valley, the area encompasses three contrast physical-geographical regions (Marynych et al., 2003) (Fig. 2). Elevated erosionally dissected loess plains with gray podzolic soils under the secondary oak-hornbeam forests and arable lands on typical chernozems represent the first region. The second region is the fragment of a floodplain and alluvial terrace with meadow soils on alluvial deposits and soddypodzolic soils on ancient alluvial deposits under meadow vegetation and pine forests, respectively. The third region within the left bank of the Dnieper includes the floodplain with soddy weakly-podzolic sandy soils under meadow vegetation and the first-third terraces with gray podzolic soils under the pine forests and typical chernozems under the arable lands.

The area harmoniously combines unique cultural and natural landscapes that has led to the consolidation and expansion of the existing protected areas and their recognition as the Key Biodiversity Area and Emerald sites (BirdLife International, 2018; Chorny, Chorna, 2013; European Environment Agency, 2017). Despite the importance of the area for the preservation of natural heritage and biodiversity, there is still the shortage of detailed information on the spatial pattern of its landscapes. In this respect, understanding of the main features of the spatial structure of environmental variables could provide a basis for field research, habitats inventory and landscape mapping necessary for the spatially precise data-driven decision making on nature conservation and land management. 


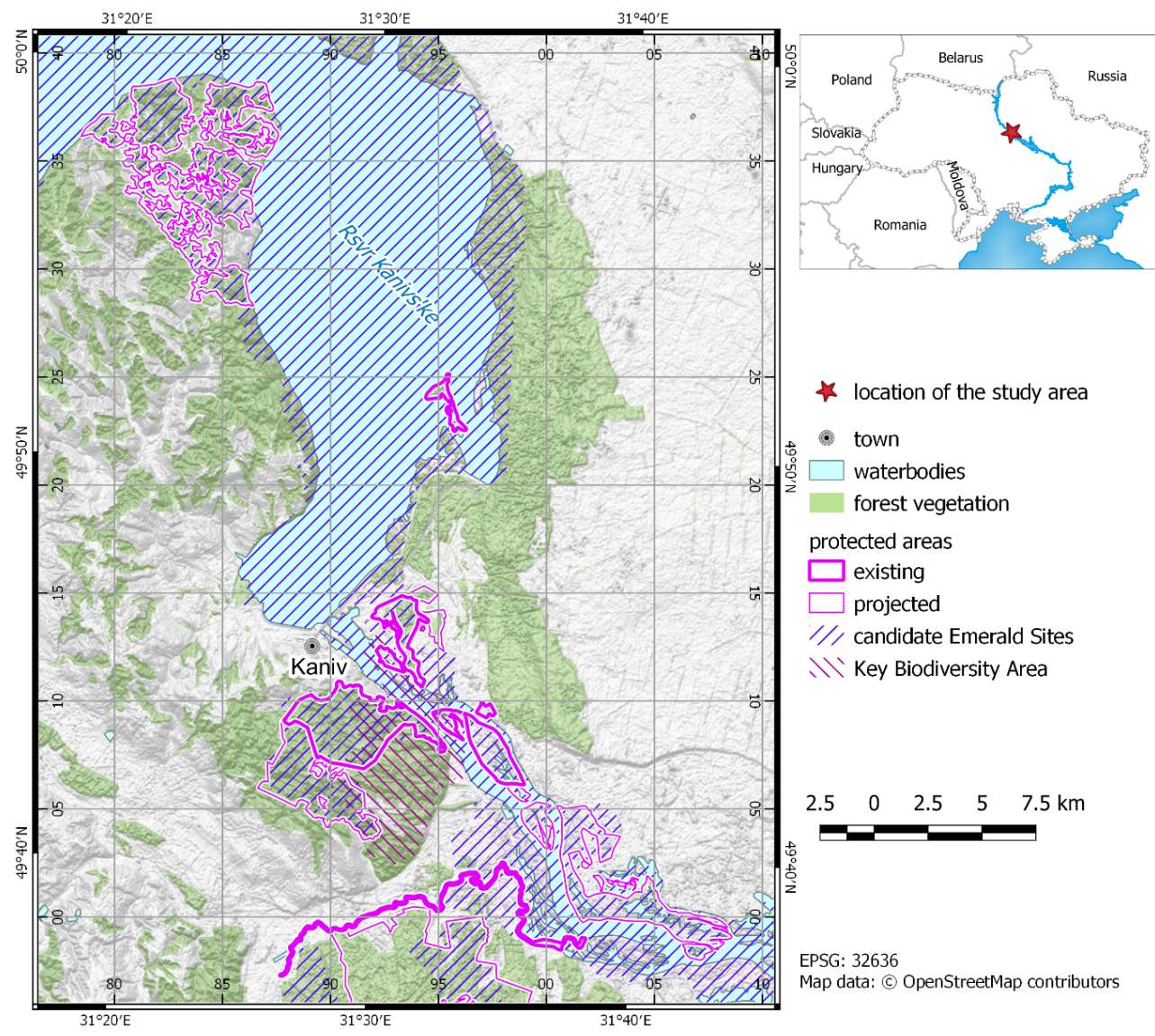

Fig. 1. Location and general view of the study area.

\section{Materials}

As landforms are an important factor of landscape heterogeneity (Swanson et al., 1988), terrain parameters composed a primary set of environmental variables. The Advanced Land Observing Satellite (ALOS) global digital surface model (DSM) 'ALOS World 3D - 30m' (AW3D30) v.2.1 released in April 2018 represented the terrain of the study area. The AW3D30 is a DSM data set with a horizontal resolution of 1 arc-second latitude and longitude mesh generated from the 5-m resolution DSM based on the images collected during 2006-2011 (Tadono et al., 2016). For the purpose of the analysis, the original DSM data were reprojected using bilinear interpolation from the geographic coordinate system WGS84 to the projected coordinate system WGS84/UTM zone $36 \mathrm{~N}$ with the resulting spatial resolution of $30 \mathrm{~m}$.

Multiple geomorphometric parameters have been developed and used to characterise the terrain variability (Hengl, Reuter, 2009; Wilson, Gallant, 2000). At the same time, Lecours et al. (2017) argued that more than 70\% of environmental variability related to the relief is captured through the limited set of the six parameters, namely, local mean, slope, local standard deviation, relative difference to mean, easterness and northerness. I derived the suggested parameters from the AW3D30 DSM in the SAGA GIS (Conrad et al., 2015) that provides the algorithms recommended by Lecours et al. (2017). 

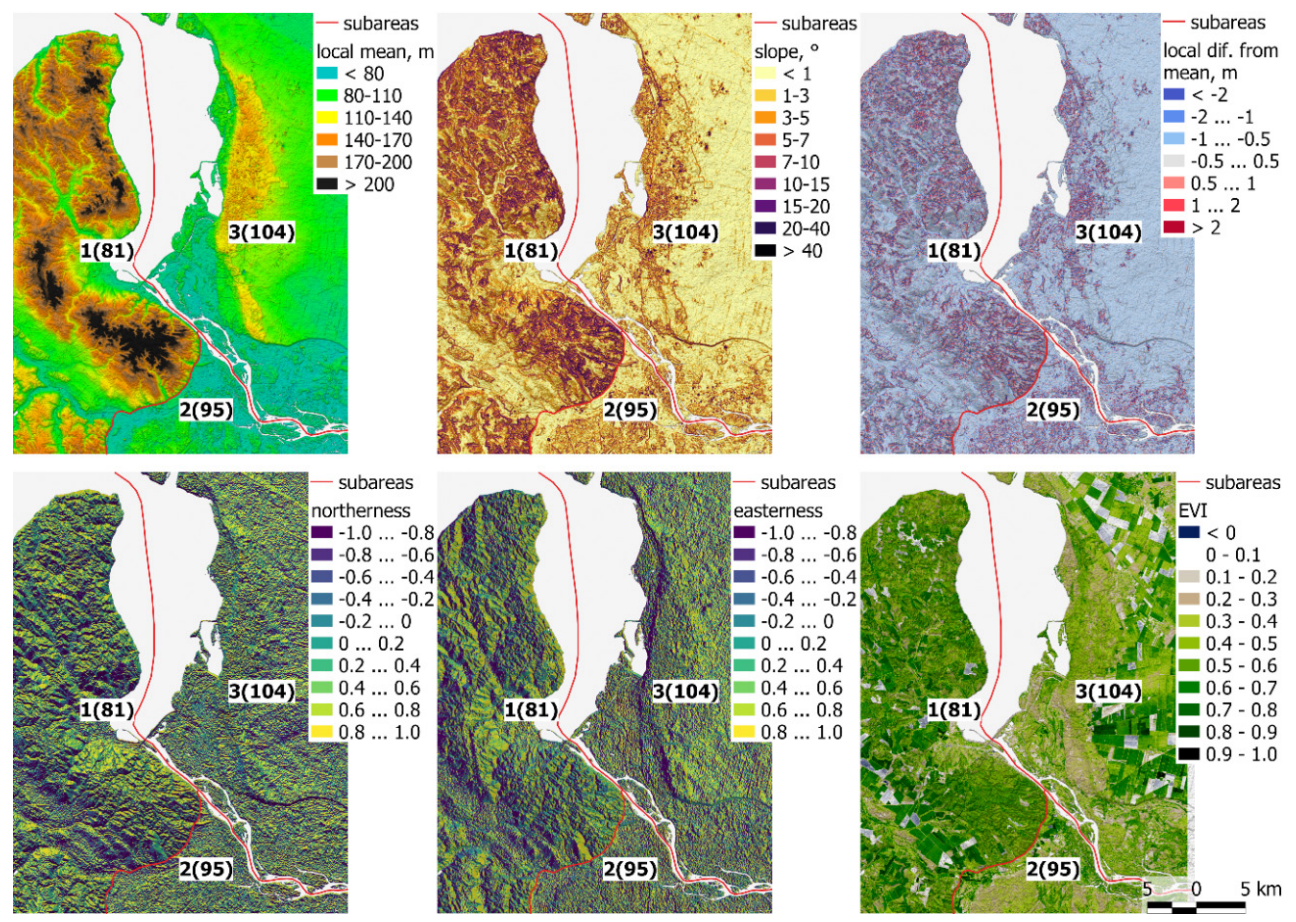

Fig. 2. Environmental variables selected for the analysis. Numbers of the sub-areas in brackets refer to the numbering scheme from Marynych et al. (2003).

The pattern of natural vegetation modified by the land-use practices is another important factor of landscape variegation. The continuous field variables of spectral vegetation indices can capture the short-term response of the vegetation to ecological and anthropogenic driving forces. For this purpose, I used the Enhanced Vegetation Index (EVI) with a spatial resolution of $10 \mathrm{~m}$ calculated from the Sentinel 2A Level 2A Bottom of Atmosphere (BOA) reflectance product of the image captured on 22 July 2017. The EVI was chosen instead of the commonly used Normalised Difference Vegetation Index because it incorporates adjustments for the canopy background and atmospheric effects, which increase its sensitivity to the vegetation signal (Huete et al., 2002).

Covariation is a common property of many environmental variables, especially of those derived from a single data source such as digital elevation model (DEM) (Graham, 2003; Lecours et al., 2017). To avoid possible data redundancy, I performed the correlation analysis to account for confounding spatial variables (Dutilleul et al., 1993; Osorio, Vallejos, 2014; R Core Team, 2017). Following the results of the correlation analysis, local standard deviation was excluded from the initial set of variables because of its strong $(0.99)$ and significant $(\mathrm{p} \leq 0.01)$ correlation with slope. The final data set included 6 environmental variables (Fig. 2).

\section{Methods}

The analysis documented as a series of scripts for the R environment (R Core Team, 2017) and based on the packages EcoGenetics (Roser et al., 2017), geoR (Ribeiro Jr., Diggle, 2016), raster (Hijmans, 2016), rgdal (Bivand et al., 2017) and sp (Bivand et al., 2013; Pebesma, Bivand, 2005). The workflow included the following steps:

1. Import of a variable's raster layer to the computing environment and calculation of the default distance of interest as the one-third of the diagonal of the raster extent. This limitation is necessary to ensure that each lag contains a sufficient number of pairs of points to produce a reliable univariate structure function value (Legendre, 
Legendre, 2012). Turner and Gardner (2015) recommended the overall linear extent of the data set to be at least twice the maximum distance (scale) to examine in order to encompass the process of a certain scale. In such a way, the distance limitation narrows down the scale of the analysis to the processes that reveal within the area of interest.

2. A random sampling of the raster by the user-defined number of points. The sample has to be limited because of computational requirements, and taking into account the distance limitation, usually 1000-3000 points are enough to produce reliable results. For the purpose of this analysis, in each case, I limited the sample size to 5000 points. The sample was exported to the ESRI Shapefile and stored as a separate data object for further analysis.

3. The calculation of the lag based on the maximum possible number of the pairs of points in the distance matrix by using the following formula:

$$
N_{\text {pairs }}=\frac{n_{\text {pixels }} \times\left(n_{\text {pixels }}-1\right)}{2}
$$

where is the total number of the pairs of points in the distance matrix and is the total number of not-null pixels in the raster.

The definition of the possible number of classes and lag distance relies on Sturges' rule:

$$
l=\frac{L}{1+3.322 \times \log _{10}\left(N_{\text {pairs }}\right)}
$$

where is a distance lag and is the maximum distance defined in the first step. The denominator of the formula defines the number of distance classes. For the convenience, the lag value is rounded to be a multiple of 5 .

On the basis of these values, the distance bins are defined by incrementing the lag by the number of classes from zero up to the maximum distance. All the results (the lag, number of classes, distance bins) are stored in intermediary data objects to be used in the following steps.

There is also a possibility for a user to manually define the lag and/or maximum distance of interest. But the simultaneous predefinition of both is not recommended because it deprives the analysis of its sense. The approach, when the lag and distance bins are based on the data properties, ensures that semivariance and autocorrelation values are calculated from the number of pairs of points that is large enough to produce reliable values. This is especially important with the limited sample size (Legendre and Legendre, 2012; Shaukat et al., 2016), which may be an issue for the sample-based analysis of the univariate structure functions of large data sets, such as rasters of continuous environmental variables.

4. Semivariance analysis using the parameters derived in the previous step (Cressie, 1993). The variograms are calculated for the nine directions from $0^{\circ}$ to $180^{\circ}$ with the increment of $22.5^{\circ}$ and tolerance angle of $11.25^{\circ}$. In addition to these directions, an omnidirectional variogram is also calculated. For better control of the validity of the values, the minimal number of pairs of points for semivariance calculation is limited to 50 (Legendre, Legendre, 2012).

Because semivariance characterises the variables with different measurement units and levels of spatial variability, it is necessary to standardise its values for meaningful compatibility and interpretation. For this purpose, I applied the approach by Rossi et al. (1992) when the values of semivariance are divided by the overall sample variance. The results are stored as a CSV-file and include the semivariance values (both original and standardised) for bins' centres, number of pairs within each bin and their standard deviation.

5. Spatial autocorrelation analysis based on Moran's I coefficient (Rosenberg, 2000). The omnidirectional and nine-directional (from $0^{\circ}$ to $180^{\circ}$ with the increment of $22.5^{\circ}$ ) correlation coefficients are calculated for the defined distance bins. The results are stored as a CSV-file and include the autocorrelation values, number of pairs within each bin, mean distance between them and p-values calculated by permutations.

Visual analytical exploration of the results relies on two-dimensional or planimetric variograms and correlograms (Legendre, Fortin, 1989; Rosenberg, 2000; Rossi et al., 1992; Wickham, 2009). The resulting polar plot consists of two parts. The top part displays the change in semivariance (or autocorrelation and its significance) values in the space of the directions from $0^{\circ}$ to $180^{\circ}$. As the plot is symmetric about its origin, the bottom $180-360^{\circ}$ part would mirror the top one. But instead of mirroring the directional values, the bottom part is used to visualise the omnidirectional values (to be identical in all directions). The combination of the directional and omnidirectional values in a single plot simplifies the exploration and understanding of the presence and features of the spatial anisotropy in the data. 


\section{Results}

The data-derived parameters calculated from the rasters by the sub-areas enable the exploration of spatial dependencies over the scales within the range from zero to the maximum distance with the grain of the lag (Table 1).

$\mathrm{T} \mathrm{a} \mathrm{b} \mathrm{le} 1$. The parameters of the univariate structure functions analysis.

\begin{tabular}{|l|c|c|c|}
\hline Variable & Lag, m & Number of distance classes & Maximum distance, $\mathbf{~ m}$ \\
\hline \multicolumn{5}{|c|}{ Sub-area 1 } \\
\hline DEM derivatives & 415 & 47 & 15355 \\
\hline EVI & 350 & Sub-area 2 & 15400 \\
\hline \multicolumn{7}{|c|}{34} & 9180 \\
\hline DEM derivatives & 270 & 39 & 8775 \\
\hline EVI & 225 & Sub-area 3 & 15580 \\
\hline \multicolumn{7}{|c|}{38} & 15750 \\
\hline DEM derivatives & 410 & 45 & \\
\hline EVI & 350 &
\end{tabular}

\section{Sub-area 1}

The local mean variable demonstrates a well-identifiable spatially anisotropic structure (Fig. 3 ). According to the variogram, the main direction of the data variability goes along the 90-270 ${ }^{\circ 1}$ axis with the contours elongating along $135-315^{\circ}$ and shrinking along $22.5-202.5^{\circ}$. Two primary semivariance peaks of 1.3 and 1.2 occur at a distance of $7 \mathrm{~km}$ (the 22.5-202.5 axis) and $11 \mathrm{~km}$ (the $157.5-337.5^{\circ}$ axis), respectively. The overall data variability reaches its maximum of 2.1 at a distance of $13 \mathrm{~km}$. The correlogram demonstrates the presence of significant positive autocorrelation up to $5 \mathrm{~km}$ with the elongation of the contours up to $8 \mathrm{~km}$ in the $146.25-326.25^{\circ}$ direction. An additional contour of zero autocorrelations appears at a distance of $12 \mathrm{~km}$.

The comparison of plots with the map of local mean suggests the presence of a two-level hierarchy in the arrangement of relief spatial structures. The variability of a finer scale is present at a distance of 5-7 km. It refers to the elevated ranges of the prevailing NW-SE orientation resulted from the dissection of hills by major elements of the erosion network. The broad-scale differences are the most clearly pronounced along the N-S direction and attribute to the landforms of a linear size of $12-13 \mathrm{~km}$, which supposed to be the hills and lowlands referring to the morphostructures formed by horsts and grabens.

Some signs of the fine-scale structure of the slope variable at a distance of less than $1 \mathrm{~km}$ present at the variogram and correlogram. The overall variability of the values pronounces in the $90-270^{\circ}$ direction and reaches its maximum of 1.7 at a distance of $15 \mathrm{~km}$. The autocorrelation

\footnotetext{
${ }^{1}$ Here and thereafter for convenience, the directional axes are given for the whole circle of $0-360^{\circ}$ because, owing to the symmetry, the bottom $\left(180-360^{\circ}\right)$ part of the plot would mirror the top $\left(0-180^{\circ}\right)$ part.
} 
values change most rapidly along the $0-180^{\circ}$ axis with the elongation of the contour of zero correlations in the latitudinal direction from $7 \mathrm{~km}$ to the distance exceeding the extent of the analysis.
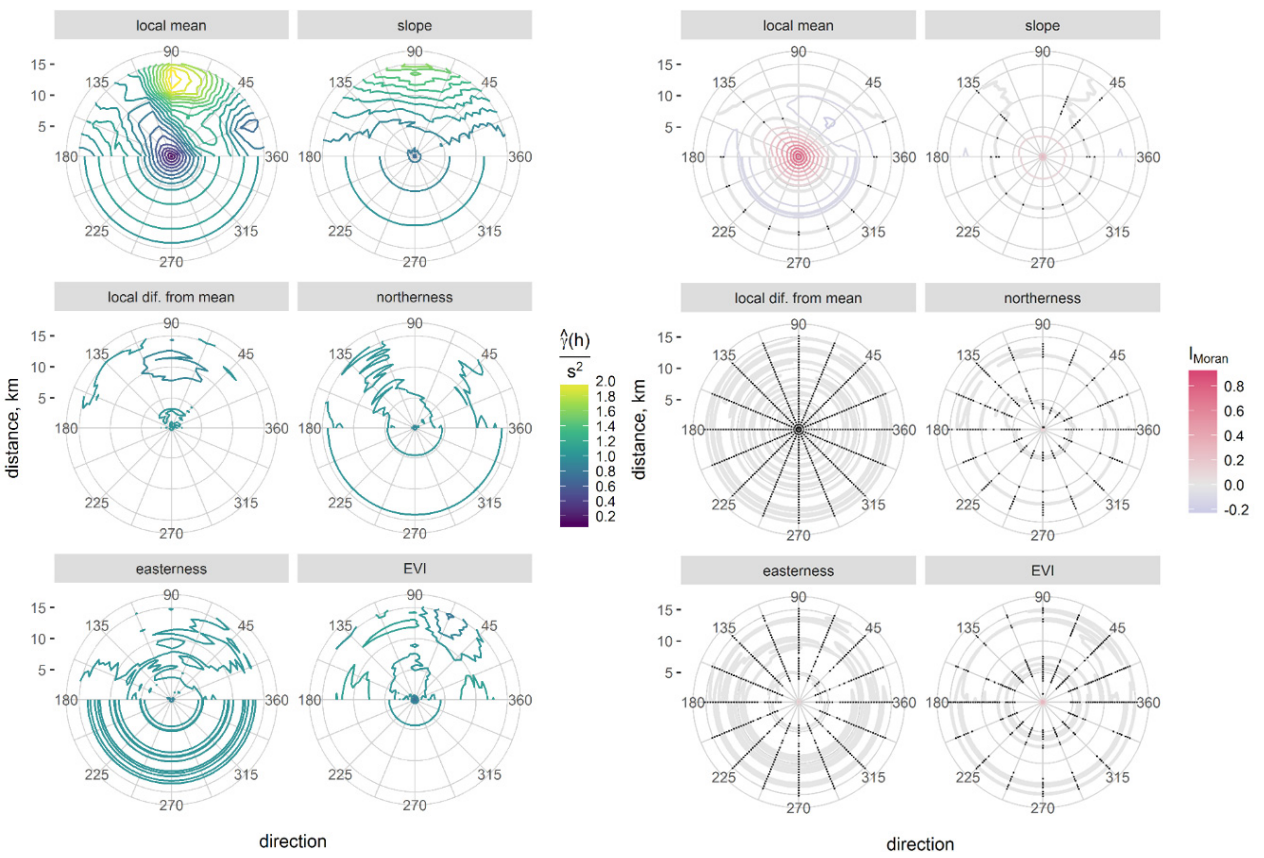

Fig. 3. The two-dimensional planimetric variograms (a) and correlograms (b) for sub-area 1. Black dots mark insignificant $(\mathrm{p}>0.01)$ autocorrelation values.

The variogram and correlogram of the local difference from mean variable demonstrate the absence of any spatially structured anisotropy with the semivariance values fluctuating around one and zero correlations of low significance.

According to the variogram, the spatial anisotropy for the northerness variable is not evident, as all semivariance values are around 1. But the correlogram demonstrates weak positive significant correlations up to a distance of $3 \mathrm{~km}$ with the elongation of the contours in the $146.25-326.25^{\circ}$ direction up to a distance of $5 \mathrm{~km}$. This is because sub-area 1 represents the elevated erosionally dissected landforms with pronounced slopes. As it was shown by the analysis of the local mean variable, the ranges of these landforms are mostly oriented along the NW-SE direction. These features are captured and reflected in the northerness spatial variability.

Similarly, for the easterness variable, there is no clear pattern on the variogram, but the correlogram demonstrates weak positive significant correlations up to a distance of $3.5 \mathrm{~km}$ (with the slight elongation of the contours along $90-270^{\circ}$ ). This is because easterness, being a direction-dependent variable, most clearly pronounces for the landforms oriented along the $\mathrm{N}-\mathrm{S}$ direction. 
The features of the EVI spatial structure are not obvious from the variogram. According to the correlogram, weak positive significant correlations persist up to $3-5 \mathrm{~km}$ with minor fluctuations, depending on the direction. This distance refers to the major size of the vegetation patches. The scale of vegetation pattern variability is, to some extent, linked to the local mean variable that may be explained by the landforms-driven distribution of land-use pattern in the area. As a result, the large patches of the vegetation (semi-natural or agricultural) coincide in their size with the size of the most prominent landforms.

\section{Sub-area 2}

The local mean variogram demonstrates the presence of a spatially anisotropic structure with the broad-scale trend of the data variability oriented along the $22.5-202.5^{\circ}$ direction and its possible maximum extended beyond the distance of interest (Fig. 4). On a finer scale, the data reach the plateau of 1 at a distance of $4 \mathrm{~km}$ with the contours elongated in the $101.25-281.25^{\circ}$ direction. The correlogram demonstrates the most rapid change in values in the $67.5-247.5^{\circ}$ direction. The contour of zero correlations is located at a distance of $4.5 \mathrm{~km}$ with fluctuations of $\pm 0.5 \mathrm{~km}$ depending on the direction. The second contour of zero correlations appears along the $135-315^{\circ}$ direction at a distance of $8 \mathrm{~km}$.
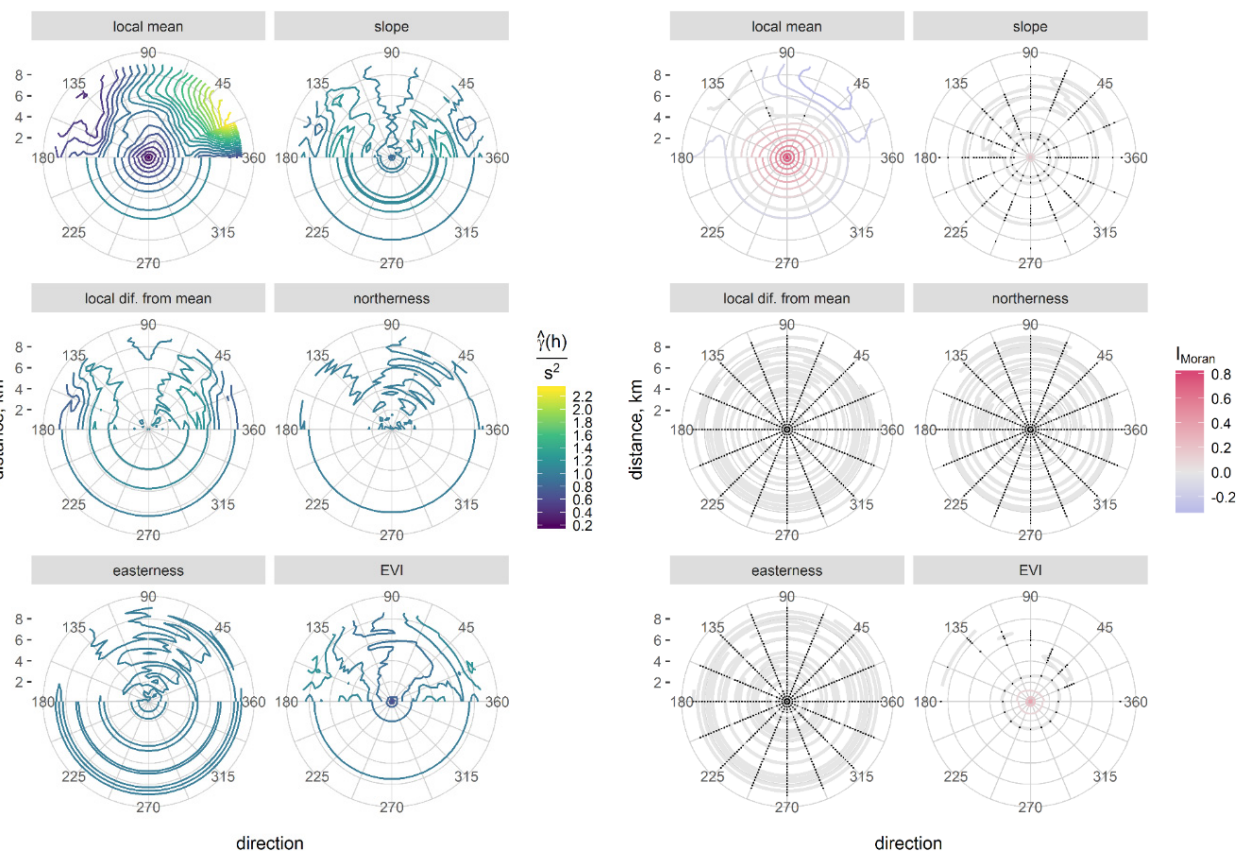

Fig. 4. The two-dimensional planimetric variograms (a) and correlograms (b) for sub-area 2. Black dots mark insignificant $(\mathrm{p}>0.01)$ autocorrelation values. 
According to the variogram, the major trend of the local mean variability oriented perpendicularly to the river valley along the ENE-WSW direction. As the trend does not reach its plateau within the distance of interest, it may identify the influence of the landform of a broader spatial scale than the area of interest (the right-bank low terrace). On a larger scale related to the landforms of a linear size of $3-4 \mathrm{~km}$, a single structural element is present. It represents an elevation in the central part of the sub-area with the slight expansion in the NNW-SSE direction and conveys not the relief but rather a perforated fragment of pine forest vegetation fixed in the AW3D30 data as an artefact. As the vegetation artefacts have not been removed from the DSM, they become especially evident in the flat areas with no prominent relief features (which is the case for sub-area 2).

The slope variogram demonstrates symmetry about the $90-270^{\circ}$ direction. Two variability maximums of 1.4 registered at a distance of $5 \mathrm{~km}$ on both the sides of the $0-180^{\circ}$ axis. The correlogram demonstrates the presence of weak positive significant correlations up to a distance of $2 \mathrm{~km}$, marked by the contour of zero correlations. This means that slope variability is symmetric in respect to $\mathrm{N}-\mathrm{S}$ direction with the most rapid changes along the NW-SE and ENE-WSW diagonals and the overall extent of data similarity of $2 \mathrm{~km}$. These features refer to the geometry and linear size of a forest vegetation-related artefact.

The variogram of the local difference from mean variable demonstrates some subtle features of a structured symmetry about $90-270^{\circ}$ direction with the moderate peaks of 1.2 at a distance of $5 \mathrm{~km}$ along the $22.5-202.5^{\circ}$ and $157.5-337.5^{\circ}$ directions. It can be assumed that overall data variability related to a general convexity-concavity pattern of the surface, which, in turn, may be attributed to the vegetation artefact fixed in the DEM. But the correlogram with zero values of low significance shows no evidence of any particular spatial structure.

The univariate structure functions of the northerness and easterness variables fluctuate around the semivariance values of one and zero correlations of low significance. This type of functions' behaviour rejects the presence of any spatial structure for these variables.

According to the EVI variogram, there is no evidence of any clearly pronounced spatial structure, because most of the values do not exceed 1 or fluctuate around it. However, two moderate peaks of 1.2 occur at a distance of $8 \mathrm{~km}$ in the $22.5-202.5^{\circ}$ and $157.5-337.5^{\circ} \mathrm{direc}-$ tions. This pattern is linked to the variograms of the slope and local difference from mean variables and reflects the shape and geometry of the vegetation artefact. The correlogram demonstrates almost spatially isotropic weak positive significant correlations up to a distance of $3 \mathrm{~km}$, marked by the contour of zero correlations. This distance refers to the generalised linear size of vegetation patches in the area.

\section{Sub-area 3}

The local mean variogram reaches its plateau at a distance of $9 \mathrm{~km}$ along the $90-270^{\circ}$ direction (Fig. 5). The directions of maximum variability of 1.5 are oriented along the $45-225^{\circ}$ and $157.5-337.5^{\circ}$ diagonals. The local mean correlogram demonstrates the presence of positive significant correlations up to a distance of $10 \mathrm{~km}$ with the general latitudinal elongation of the contours. The fragments of the secondary contours of zero correlations appear in the longitudinal direction at a distance of $13 \mathrm{~km}$. 

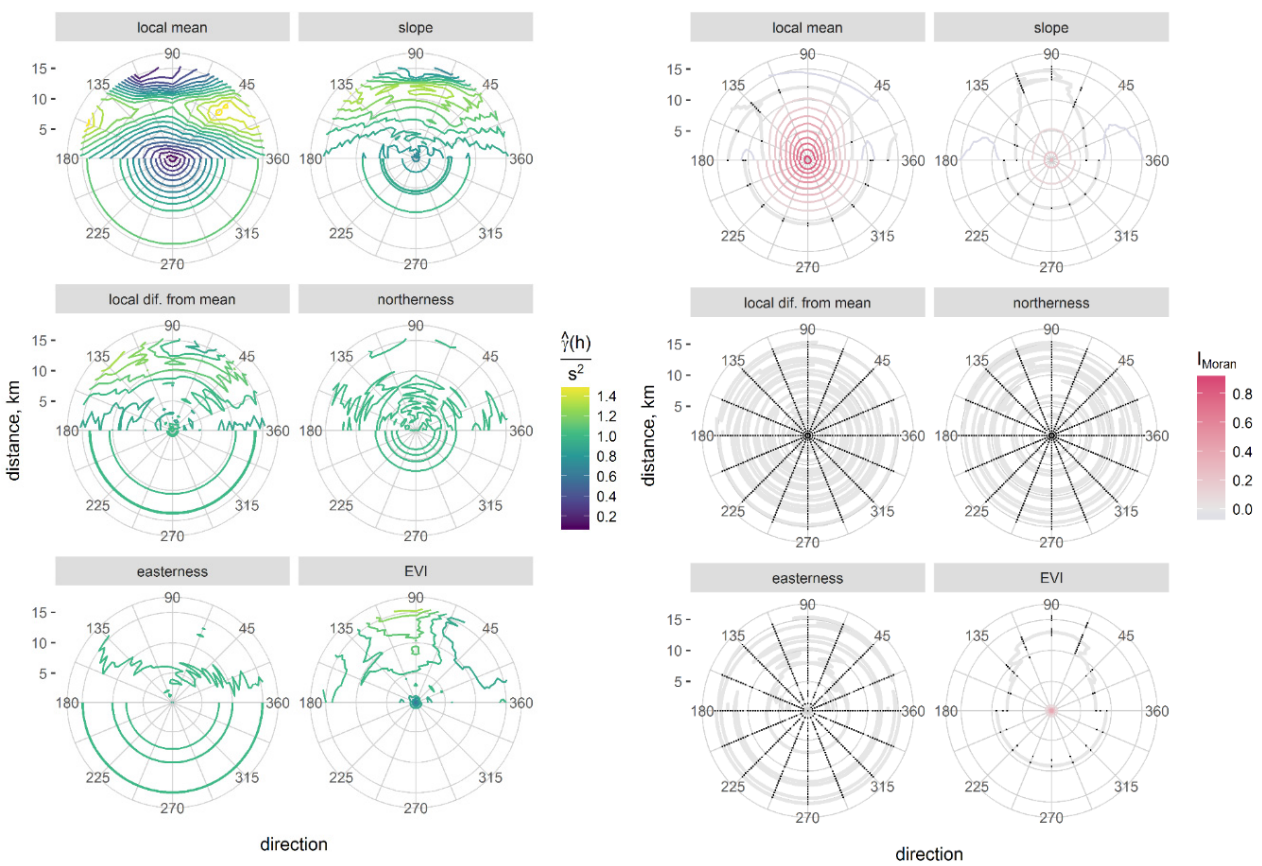

Fig. 5. The two-dimensional planimetric variograms (a) and correlograms (b) for sub-area 3. Black dots mark insignificant $(\mathrm{p}>0.01)$ autocorrelation values.

According to the variogram and correlogram, within sub-area 3, the relief of the area demonstrates low variability with the main spatial structures latitudinally oriented along the river valley and the most rapid changes occurring along the NE-SW and WNW-ESE directions. Comparing this information with the map, it can be concluded that major landforms responsible for the differentiation are the floodplain and first, second and the fragment of the third terraces of the Dnieper clearly identifiable in the relief.

According to the slope variogram, the main direction of the data variability orients along the $135-315^{\circ}$ direction and reaches its maximum of 1.4 at a distance of $15 \mathrm{~km}$. The overall plateau of the variogram is most clearly pronounced in the latitudinal direction at a distance of $12 \mathrm{~km}$. At the correlogram, the contour of zero correlations elongates latitudinally and passes at distances from 6 to $13 \mathrm{~km}$ depending on the direction. Within sub-area 3, the slope variable is most clearly linked to the variable of local mean. This is because the main changes in the slope values are strictly related to the curbs and ledges of the river terraces.

The variogram of local difference from mean demonstrates some resemblance of its contours pattern to the slope variogram. The trend of the maximum variability lies along the $135-315^{\circ}(\mathrm{NW}-\mathrm{SE})$ direction. A possible explanation for this is that the distribution of noise and artefacts registered by local difference from mean in this sub-area related to the distribution pattern of vegetation patches (forest and agricultural), which in turn coincide with the 
major landforms. At the same time, the correlogram does not reveal any pattern and presents close to zero correlation values of low significance.

The semivariance values of northerness and easterness fluctuate around 1; correlograms demonstrate zero correlation values of low significance. The univariate structure functions do not confirm the presence of any spatial structure within sub-area 3 for the variables of northerness and easterness.

The EVI variogram demonstrates the major trend of the data variability oriented along the $90-270^{\circ}$ direction and reaches its maximum of 1.3 at a distance of $15 \mathrm{~km}$ with a possible plateau probably exceeding the distance of interest. At the correlogram, the contour of zero correlations elongates latitudinally and passes at distances from 7 to $13 \mathrm{~km}$ depending on the direction. For this sub-area, the driving forces of vegetation pattern are tracked at two levels. At the first level, there is a fine-scale variability within a distance of $1 \mathrm{~km}$ related to the internal heterogeneity of vegetation patches such as individual agricultural fields, meadows and forests. At the second level, these are broad-scale patterns explained by overall land-use differences: the areas mostly dominated by the agricultural vegetation as opposed to the areas under (semi)natural vegetation. At this level, the EVI plots demonstrate some resemblance to the plots of local mean, slope and local difference from mean. This is because the distribution of land-use pattern in the area coincides with the major landforms. The floodplain and first terrace are covered with meadows, the second terrace is under agricultural land use and pine forest grows on the fragments of the third terrace.

\section{Discussion}

At a given scale of the analysis, not all environmental variables turned out to be equally responsible for the presence of a spatial pattern and informative for its explanation within the study area. The local mean variable within each sub-area demonstrates a clearly identifiable spatial structure with the obvious features of spatial anisotropy. Comparison of the distances and directions of the maximum semivariance and zero autocorrelations with the linear size and orientation of the landforms allowed assuming the scale of the landforms, which define the major features of the spatial structure of the area as well as their linkages to the other variables. For sub-area 1, these are the NW-SE oriented elevated ranges with a length of 5-7 $\mathrm{km}$. For sub-area 3, these are latitudinally oriented structural elements of the river valley (the floodplain and terraces). In both the cases, the vegetation pattern demonstrates linkages with the relief because the spatial distribution of the land use tends to follow the major landforms.

The other DEM derivatives are less informative. The pattern of the slope variable spatial anisotropy usually inherits small-scale features of the local mean variable. The variable of local difference from mean for all sub-areas, excluding sub-area 2, demonstrates a type of behaviour close to a random variable characterised by the flat variograms (the values fluctuating around 1$)$ and zero correlations of low significance $(p>0.01)$. Similarly, the variables of northerness and easterness behave randomly, excluding sub-area 1.

The random type of behaviour symbolises the absence of any detectable spatial trends or patterns and can be attributed to two reasons. First, the structure may reveal itself at a much larger scale being registered at the scale of the analysis as a random noise. Second, the de- 
rivatives of an initial surface are prone to be affected by the noise represented by speckle and artefacts in the original data, which make the spatial structure less detectable.

In addition, in flat areas, vegetation tends to be registered in the DEM as artefacts. This primarily affects the resulting values of slope and local difference from mean, which characterise the shape of a surface (steepness, convexity-concavity). Because this shape reflects a vegetation pattern, the variograms and correlograms of slope, local difference from mean and EVI may demonstrate resemblance, as within sub-areas 2 and 3.

Nevertheless, even if the variables demonstrate a weakly detectable structure, the results of the analysis usually suggest the potential distances of interests at a larger scale, similar to that in the case of EVI for all the sub-areas or northerness/easterness within sub-area 1 . This prevents arbitrary decisions and multiple tests and trials for choosing the parameters for a further detailed exploration.

Variograms and correlograms of a single variable usually demonstrate the overall resemblance accompanied by some discrepancies in the directions and characteristic distances of the contours' expansion. These discrepancies could make interpretation more difficult. Rossi et al. (1992) argued that it is necessary to jointly interpret variograms and correlograms because they differently account for local variability and thus highlight different aspects of the data. Variograms do not filter out lag means and variances, so they reveal a lag-to-lag local variability and underline the dissimilarity of values. Correlograms, on the contrary, account for regional patterns because of filtering out local variability and accentuate similarity in values. Also, because variograms do not filter out a large-scale variability, they tend to overestimate characteristic scales of spatial anisotropy. These differences partially explain the dissimilarities of variograms against correlograms.

Another part of the explanation attributed to the differences between the algorithms in definition and calculation of the direction. Variograms are calculated with the $\pm 11.25^{\circ}$ directional tolerance, which slightly blurs the resulting values (Cressie, 1993; Ribeiro Jr., Diggle, 2016). The calculation of correlograms involves the weighing of distance matrix elements based on their association with a certain fixed direction (Rosenberg, 2000; Roser et al., 2017). These features of the algorithms may also add up to the inconsistencies in patterns.

\section{Conclusion}

Comparing to conventional single-dimensional omnidirectional univariate structure functions, directional functions conveyed through an appropriate visualisation identify main features of spatial anisotropy of environmental variables. Joint exploration of directional correlograms and variograms allows for the confirmation or rejection of the existence of a spatial pattern and, in the case of its presence, to describe its spatial anisotropy; to quantify the data variability range, the scale(s) of maximum variances and zero correlations; and to check for the links between variables. These characteristics help to identify main features of spatial variability and its scales, to investigate the dissimilarities in the spatial patterns related to the processes of different scale and to hypothesise on the underlying factors and/or processes responsible for landscape heterogeneity at a certain scale.

In the case of absence of clearly identifiable spatial anisotropy, the analysis based on the data-derived parameters should be considered as an exploratory technique. Its results can 
be applied to guide further investigation and to identify informative scales and distances of potential interest.

For DEM derivatives, which are prone to be distorted by noise and artefacts, the quality of the data affected the ability to identify large-scale spatial patterns. This question requires further investigation in the context of both DEM preprocessing techniques (Gallant et al., Dowling, 2012) and the potential to use newly available more detailed sources of elevation data (Grohmann, 2018).

\section{Acknowledgements}

I thank Professor Mykhailo Grodzinskyi for comments that improved an earlier version of this manuscript. I also thank Copernicus Research and User Support (RUS) Service for providing the computing environment to process and analyse the data.

\section{Supplementary material}

The data sets analysed and generated during the current study, codes and full-size figures associated with this article are available in the Open Science Framework repository at https://osf.io/3hfq6.

\section{References}

BirdLife International (2018). The world database of key biodiversity areas. http://www.keybiodiversityareas.org Bivand, R., Keitt, T. \& Rowlingson B. (2017). rgdal: Bindings for the geospatial data abstraction library. https://cran.rproject.org/package $=$ rgdal

Bivand, R.S., Pebesma, E. \& Gomez-Rubio V. (2013). Applied spatial data analysis with R. New York: Springer. http:// www.asdar-book.org/

Chorny, M.G. \& Chorna L.O. (2013). Kaniv Nature Reserve: preconditions of foundation, retrospective analysis of activities, current status and development perspectives (in Ukrainian). Kiev: Taras Shevchenko National University.

Conrad, O., Bechtel, B., Bock, M., Dietrich, H., Fischer, E., Gerlitz, L., Wehberg, J., Wichmann, V. \& Böhner J. (2015). System for Automated Geoscientific Analyses (SAGA) v. 2.1.4. Geoscientific Model Development, 8(7), 1991-2007. DOI: 10.5194/gmd-8-1991-2015.

Copernicus Programme (2015). N2K 2012 - Copernicus Land Monitoring Service. https://land.copernicus.eu/local/ natura/natura-2000-2012?tab=metadata

Cressie, N.A.C. (1993). Statistics for spatial data. Hoboken: John Wiley \& Sons, Inc. DOI: 10.1002/9781119115151.

Dutilleul, P., Clifford, P., Richardson, S. \& Hemon D. (1993). Modifying the $t$ test for assessing the correlation between two spatial processes. Biometrics, 49(1), 305. DOI: 10.2307/2532625.

European Environment Agency (2017). Emeral Network - General Viewer. http://emerald.eea.europa.eu/

Gallant, J.C., Read, A.M. \& Dowling T.I. (2012). Removal of tree offsets from SRTM and other digital surface models. ISPRS - International Archives of the Photogrammetry, Remote Sensing and Spatial Information Sciences, 39(B4), 275-280. DOI: 10.5194/isprsarchives-XXXIX-B4-275-2012.

Graham, M.H. (2003). Confronting multicollinearity in ecological multiple regression. Ecology, 84(11), 2809-2815. DOI: $10.1890 / 02-3114$.

Grohmann, C.H. (2018). Evaluation of TanDEM-X DEMs on selected Brazilian sites: Comparison with SRTM, ASTER GDEM and ALOS AW3D30. Remote Sens. Environ., 212, 121-133. DOI: 10.1016/j.rse.2018.04.043.

Hansen, A.J. \& DeFries R. (2007). Ecological mechanisms linking protected areas to surrounding lands. Ecol. Appl., 17(4), 974-988. DOI: 10.1890/05-1098.

Hengl, T. \& Reuter H.I. (Eds.) (2009). Geomorphometry: concepts, software, applications. Developments in Soil Science, 33. Amsterdam: Elsevier.

Hijmans, R.J. (2016). raster: Geographic data analysis and modeling. https://cran.r-project.org/package=raster

Huete, A., Didan, K., Miura, T., Rodriguez, E., Gao, X. \& Ferreira L.(2002). Overview of the radiometric and biophysical performance of the MODIS vegetation indices. Remote Sens. Environ., 83(1-2), 195-213. DOI: $10.1016 / \mathrm{S} 0034-4257(02) 00096-2$. 
Lecours, V., Devillers, R., Simms, A.E., Lucieer, V.L. \& Brown C.J. (2017). Towards a framework for terrain attribute selection in environmental studies. Environmental Modelling and Software, 89, 19-30. DOI: 10.1016/j.envsoft.2016.11.027.

Legendre, P. \& Fortin M.J. (1989). Spatial pattern and ecological analysis. Vegetatio, 80(2), 107-138. DOI: 10.1007/ BF00048036.

Legendre, P. \& Legendre L. (2012). Numerical ecology. Elsevier.

Marynych, O.M., Parkhomenko, G.O., Petrenko, O.M. \& Shishchenko P.G. (2003). The improved scheme of the physical-geographical zoning of Ukraine (in Ukrainian). Ukrainian Geographical Journal, 1, 16-21.

Osorio, F. \& Vallejos R. (2014). SpatialPack: Package for analysis of spatial data. http://cran.r-project.org/ package $=$ SpatialPack

Pebesma, E.J. \& Bivand R.S. (2005). Classes and methods for spatial data in R. R News, 5(2), 9-13. https://cran.rproject.org/doc/Rnews/

R Core Team (2017). R: A language and environment for statistical computing. Vienna: R Foundation for Statistical Computing. https://www.r-project.org/

Ribeiro,P.J. Jr. \& Diggle P.J. (2016). geoR: Analysis of geostatistical data. https://cran.r-project.org/package=geoR

Rosenberg, M.S. (2000). The bearing correlogram: a new method of analyzing directional spatial autocorrelation. Geographical Analysis, 32(3), 267-278. DOI: 10.1111/j.1538-4632.2000.tb00428.x.

Roser, L., Vilardi, J., Saidman, B. \& Ferreyra L. (2017). EcoGenetics: Spatial analysis of phenotypic, genotypic and environmental data. https://cran.r-project.org/package=EcoGenetics

Rossi, R.E., Mulla, D.J., Journel, A.G. \& Franz E.H. (1992). Geostatistical tools for modeling and interpreting ecological spatial dependence. Ecol. Monogr., 62(2), 277-314. DOI: 10.2307/2937096.

Shaukat, S.S., Rao, T.A. \& Khan M.A. (2016). Impact of sample size on principal component analysis ordination of an environmental data set: effects on eigenstructure. Ekológia (Bratislava), 35(2), 173-190. DOI: 10.1515/ eko-2016-0014.

Swanson, F.J., Kratz, T.K., Caine, N. \& Woodmansee R.G. (1988). Landform effects on ecosystem patterns and processes. BioScience, 38(2), 92-98. DOI: 10.2307/1310614.

Tadono, T., Nagai, H., Ishida, H., Oda, F., Naito, S., Minakawa, K. \& Iwamoto H. (2016). Generation of the $30 \mathrm{~m}-\mathrm{mesh}$ global digital surface model by ALOS PRISM. International Archives of the Photogrammetry, Remote Sensing and Spatial Information Sciences - ISPRS Archives, 41, 157-162. DOI: 10.5194/isprsarchives-XLI-B4-157-2016.

Turner, M.G. \& Gardner R.H. (2015). Landscape ecology in theory and practice. New York: Springer. DOI: 10.1007/978-1-4939-2794-4.

Wickham, H. (2009). ggplot2: Elegant graphics for data analysis. New York: Springer-Verlag. http://ggplot2.org Wilson, J.P. \& Gallant J.C. (Eds.) (2000). Terrain analysis: Principles and applications. New York: Wiley. 